\title{
LARGE SCALE DISCRETE ELEMENT SIMULATION CAMPAIGNS - SIMULATING EXTRATERRESTRIAL SOILS IN PARTSIVAL
}

\author{
Roy Lichtenheldt ${ }^{1}$, Shoko Ono ${ }^{1,2}$ and Leon Stubbig ${ }^{1}$ \\ ${ }^{1}$ Institute of System Dynamics and Control - German Aerospace Center (DLR) \\ Münchener Straße 20, 82234 Weßling, Germany \\ Roy.Lichtenheldt@dlr.de,www.dlr.de/sr \\ ${ }^{2}$ Department of Aerospace Engineering, Tohoku University \\ Aoba 6-6-01, Sendai, 980-8579, Japan
}

Key words: Granular Materials, DEM, Contact Problems, Large Scale

\begin{abstract}
In planetary exploration, testing under the actual mission conditions is inherently not possible. Hence, simulation campaigns complement ground test campaigns. This specially applies to surface missions that include the complex behaviour of soils under non-terrestrial gravitation. Increasingly ambitious mission goals made large simulation campaigns with very precise particle models necessary for the simulation of soil interaction. Thus, to limit the amount of time and the computation hardware needed, DLR developed the particle simulation tool "Sir partsival". This tool does not only speed up simulations by usage of GPU computing, but also integrates the institute's experience in modelling of soil on Earth and beyond. Using partsival it was possible to speed up simulations by more than a factor of ten and thus conduct large simulation campaigns. Two examples are shown: a large, on-going validation campaign of DEM for wheel simulations, and the completed traction optimization for the MMX rover wheel.
\end{abstract}

\section{INTRODUCTION}

Large scale simulation campaigns occur increasingly often in industry scale applications. Especially in planetary exploration, such campaigns help to lower uncertainty due to lacking test possibilities, e.g. due to different gravitation. However, with lower cost missions in planetary exploration, development time is significantly shortened, too. This fact raises problems for large scale simulations and campaigns using the Discrete Element Method (DEM), which is inherently computationally expensive due to the high number of repeated neighborhood checks. To address this issue, DLR developed the particle simulation tool "Sir partsival" [1]. This tool does not only use GPU technology to speed simulations up, but also inherits special modeling features and engineering assumptions to allow for large scale campaigns and simulations even within tight schedules.

In the paper, some of partsival's special features will be described: 1) The models for "tilting spheres" [2], a mathematical approach of covering the rolling behaviour of complexly shaped grains by spherical contact bodies. 2) The microphysics-based approach for cohesion modelling. 3) partsival's main integration scheme is a special semi-implicit scheme based on a jolt-prediction [3] in order to eliminate contact detection steps in predictor-corrector iterations. Moreover, considerations on numerical stability, step size control as well as accuracy will be given. Finally, recent validation efforts and applications are presented. 


\section{STATE OF THE ART}

DEM has been developed since the first publication by Cundall and Strack in 1979 [4]. This section gives an overview of the method and its application in Terramechanics.

\subsection{Discrete Element Method}

The flow and bulk behavior of granular materials is still not well understood and lacks constitutive equations derived from first principles. Therefore, the field of Terramechanics has made use of experiments and empirically derived relations. These methods are not well suited for the investigation of extra-terrestrial problems, where the varying gravity levels make experimentation opportunities scarce or impossible. Thus, there has been a push for better numerical simulation methods in recent years. These modeling techniques range from improved (semi-) empirical models models [5], over continuum methods, to particle methods such as the Discrete Element Method [6] (listed in the order of increasing fidelity and computational effort). DEM has characteristics that make it particularly suited for detailed simulation of tool-soil interaction: It implicitly covers large soil deformations and allows for inspection of the soil flow around tool features such as wheel grousers. DEM also makes fewer assumptions than empirical methods with most parameters being derived from physical quantities [7]. In DEM, a granular medium is represented by an assembly of particles that interact based on contact laws. A simulation then consists of three main steps: contact detection, force evaluation and integration of the equations of motion.

The locomotion problems experienced by NASA's MER rovers on soft Martian soil further drove simu-

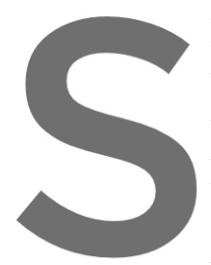

lation efforts [6, 8]. Focusing on NASA's in Sight Mission and wheeled roversas applicafions, [9] builds

the theoretical basis for partsival. Recent work at DLR includes stu

tion up slopes in Martian gravity [10] and the de

Phobos [11]. Furthermore [12] shows the influence

and discusses solutions

partsival has been developed with Terramechanics
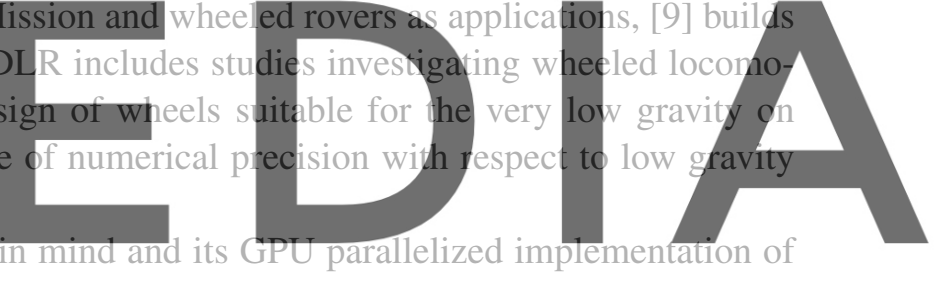

soft-sphere DEM is well suited for simulations in this area

Register for free at https//www.scipedia.com to download the version without the watermark

\subsection{GPU Computing}

Modern GPUs allow for massively parallel computation. In partsival as many particles as shader units can be processed at once. Dependent on the GPU architecture these particles are computed in lock-step creating problems when interparticle communication on values is required. Furthermore, gaming cards feature very good performance in single floating point precision but are very slow in double precision. However, both gaming and industrial grade GPUs have their share in DEM simulations, as not every simulation requires double precision as will be shown later. In terms of the the GPU framework, partsival is set up around OpenGL compute shaders [1].

One of partsival's main ideas is that communication between GPU and CPU, commonly done over slower PCI-E x16 interfaces, shall be omitted whenever possible. Thus in partsival, particles "live" in the GPU space only and are only copied to CPU-space when data is stored to hard drives. All the physics are thus done on the GPU itself. This enables partsival to be faster than commercial CPU DEM codes by a factor of ten or larger, see Figure 1. Given this performance gain, partsival is a tool especially suitable for the large scale campaigns at hand. 


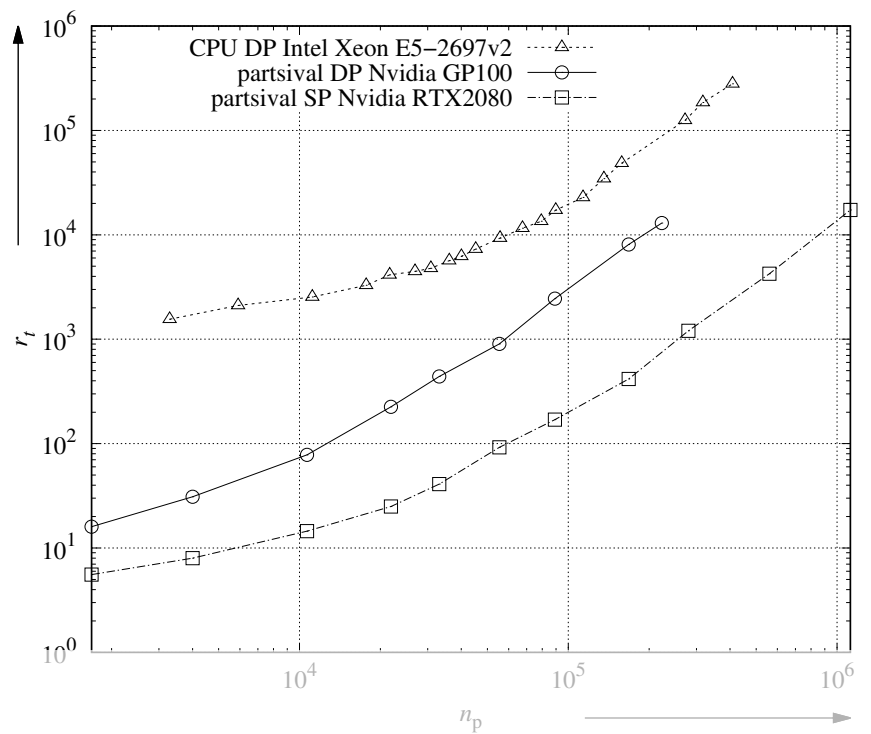

Figure 1: Performance comparison of partsival on different single GPUs in different floating point precision with respect to parallel CPU code using up to 48 CPU cores [1]

3 CONTACT MODELING

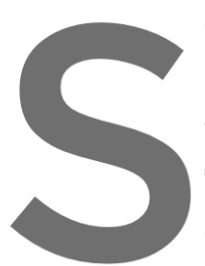

Contact models govern

3.1 Basic Contact Model

The basic contact model

directions as well as a roll
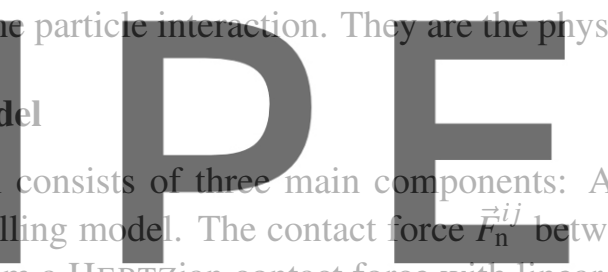

direction is computed from a HERTzian contact force with linear damping [9]:
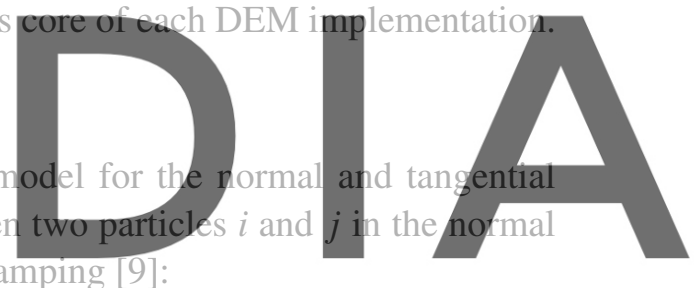

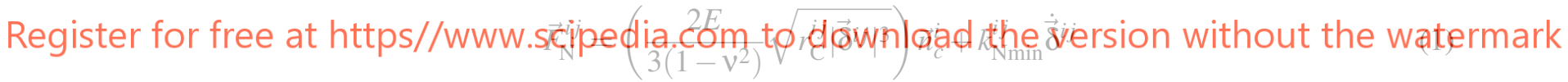

where $E$ is Young's modulus, $v$ is PoISSON ratio, $r_{\mathrm{c}}=0.5\left(r_{i}+r_{j}\right)$ is the contact radius, $\delta_{n}^{i j}$ is the overlap in normal direction, $\rho_{\mathrm{p}}$ is density and $k_{\mathrm{Nmin}}$ is the damping coefficient.

In the tangential direction, stick-slip friction is implemented: The particle is sticking if the relative tangential velocity of two contacting particles $\dot{\vec{\delta}}_{\mathrm{T}}^{i j}$ is smaller than the cut-off velocity $v_{\mathrm{Tmin}}$ and the tangential regularization force $F_{\mathrm{cT}}^{i j}$ is lower than the sticking friction limit. As sticking features elastic deformations in reality, a KELVIN element is used to regularize this state. If $F_{\mathrm{cT}}^{i j}$ exceeds the sticking limit, the particle starts slipping. Summing up the frictional forces are applied as follows [13]:

$$
\begin{array}{r}
\vec{F}_{c \mathrm{~T}}^{i j}=c_{\mathrm{T}}^{i j} \cdot \vec{\delta}_{\mathrm{T}}^{i j} \cdot \operatorname{sign}\left(\vec{\delta}_{\mathrm{T}}^{i j} \cdot \dot{\vec{\delta}}_{\mathrm{T}}^{i j}\right)+k_{\mathrm{T}}^{i j} \cdot \dot{\vec{\delta}}_{\mathrm{T}}^{i j} ; \quad \vec{F}_{\mathrm{hT}}^{i j}=\left|\vec{F}_{\mathrm{N}}^{i j}\right| \cdot \tan \left(\phi_{\mathrm{h}}\right) \\
\vec{F}_{\mathrm{T}}^{i j}= \begin{cases}\vec{F}_{c \mathrm{~T}}^{i j} & \forall\left|\vec{F}_{c \mathrm{~T}}^{i j}\right| \leq \vec{F}_{\mathrm{hT}}^{i j} \wedge\left|\dot{\vec{\delta}}_{\mathrm{T}}^{i j}\right| \leq v_{\mathrm{Tmin}}^{i j} \\
\left|\vec{F}_{\mathrm{N}}^{i j}\right| \cdot \tan \left(\phi_{\mathrm{g}}\right) \cdot\left(\dot{\vec{\delta}}_{\mathrm{T}}^{i j}\right)_{0} \quad \forall\left|\vec{F}_{c \mathrm{~T}}^{i j}\right|>\vec{F}_{\mathrm{hT}}^{i j} \vee\left|\dot{\vec{\delta}}_{\mathrm{T}}^{i j}\right|>v_{\mathrm{Tmin}}^{i j}\end{cases}
\end{array}
$$

where $k_{\mathrm{T}}$ is the tangential damping coefficient and $\phi_{\mathrm{g}}$ and $\phi_{\mathrm{h}}$ are slipping and sticking angle of friction, respectively. Previous DEM studies showed problems with excessive particle rolling which led to the 


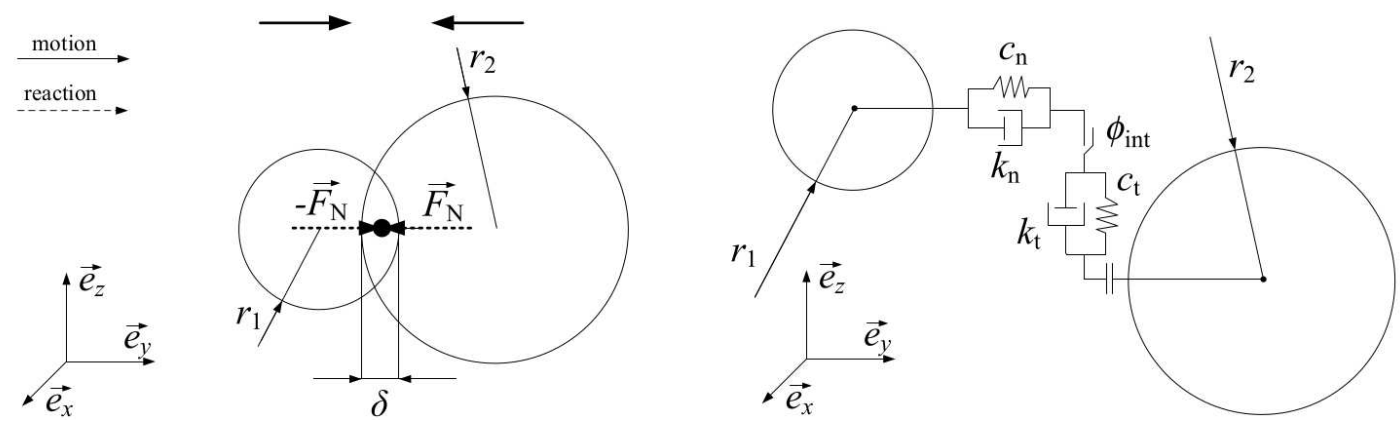

Figure 2: The contact models in normal and tangential directions $[9,13]$

introduction of an additional rolling resistance model [14]. This resistance torque is implemented in partsival as a basic rolling model.

\subsection{Tilting Models}

Basic resistance torque models like [14] are usually empirical and do not cover micromechanical rolling behaviour. In partsival, the approach from [2] is used to cover tilting behaviour without sacrificing computation performance. Thereby, angular but regular rotation shapes are mapped to the spherical particles. This yields non-continuous torques that dependent on tangential as well as normal forces, whereas their ratio together with the current rotation angle around a single axes determine the torque and its direction. Hence, these to polygonal shapes as well arm lengths are calculated shape's edge. The detaile ratio angle $\gamma_{i}$, is given
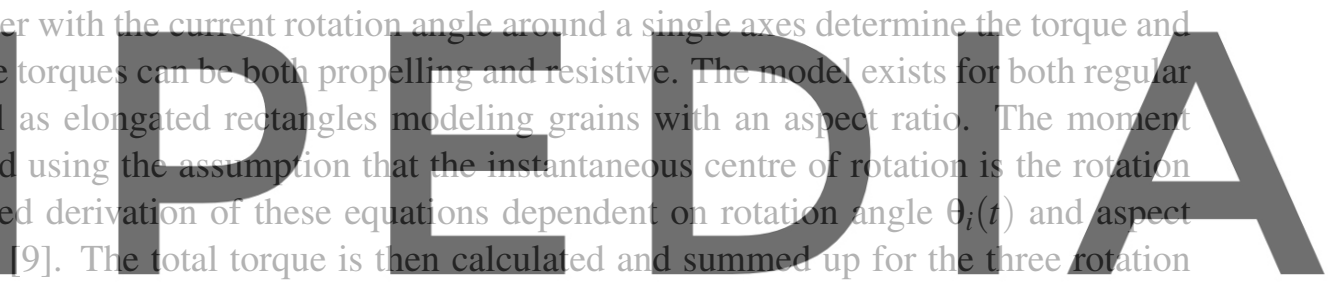
planes $\underline{E}_{\mathrm{R}}^{j k}$ as $[2,9]$ :

Register for free at https//wyww.scipedia.com to download the version without the watermark

$$
\begin{gathered}
\vec{M}_{\mathrm{RG}}=\sum_{i, j, k=1}\left(-\left[l_{\mathrm{T}}^{j k}\left(\theta_{i}(t), \gamma_{i}\right) \cdot \vec{N}_{\mathrm{c}}^{j k}\right] \times \vec{F}_{\mathrm{R}}^{j k}-\left[l_{\mathrm{N}}^{j k}\left(\theta_{i}(t), \gamma_{i}\right) \cdot \vec{T}_{\mathrm{c}}^{j k}\right] \times \vec{F}_{\mathrm{R}}^{j k}\right) \cdot \varepsilon_{i j k}^{2} \\
i, j, k \in\{1,2,3\}_{\mathbb{N}} \wedge \boldsymbol{\varepsilon}_{i j k} \neq 0
\end{gathered}
$$

with $\vec{F}_{\mathrm{R}}^{j k}$ the particle's total force projected in the rotation plane and $\varepsilon_{i j k}$ is the LEVI-CIVITA symbol.

\subsection{Cohesion Modeling}

For soils containing portions of water (pseudo-cohesion) or with sufficiently small grains, attractive forces between grains cause cohesion stresses. In low gravity environments, these forces get increasingly important as frictional forces decrease with gravity, while attractive force remain constant. Hence, under sufficiently low gravity, cohesion forces can become the governing force between particles. In partsival these forces are modelled by their physical micro-scale origin. Three forces have been identified in decreasing order of significance: VAN DER WAALS force $F_{\mathrm{VdW}}$, electrostatic force $F_{\mathrm{e}}$ and self gravitation $F_{\mathrm{G}}$. In [15], these forces have been derived and implemented as a total cohesion force in partsival: 


$$
\vec{F}_{\mathrm{c}}=\left(\left(\frac{Q_{\mathrm{i}}}{16 \cdot \pi}+\frac{\pi}{2} \varepsilon \cdot C_{\mathrm{i}} \cdot U_{\mathrm{c}}^{2}\right) \cdot\left(\frac{r_{\mathrm{g}}}{2 \cdot R+a_{0}}+\frac{n_{R} \cdot R}{a_{0}^{2}}\right)+G \frac{m_{i} \cdot m_{j}}{a_{0}+2 \cdot R+2 \cdot r_{\mathrm{g}}} \cdot\right) \cdot \frac{r_{\mathrm{p}}^{2}}{r_{\mathrm{g}}^{2}} \cdot \vec{n}_{c}
$$

with $Q_{\mathrm{i}}$ the interaction energy between the grains, $a_{0}$ the grain distance including roughness effects, $R$ the roughness itself, $n_{R}$ the number of contacts, $\varepsilon$ the dielectric constant, $C_{\mathrm{i}}$ the influence constant, $U_{\mathrm{c}}$ the contact potential, $m_{i, j}$ the particle masses and $G$ the gravitational constant. The equation shows that these forces are all dependent on the grain size $r_{\mathrm{g}}$, but with different exponents. Using upscaling laws for particle size, as common in DEM, would thus cause wrong orders of magnitudes of these forces. Hence, the real material grain sizes are used for the cohesion calculation and the cohesional stresses between grains are computed. These stresses are applied to the larger particles, mimicking portions of grains.

\section{PERFORMANCE IMPROVEMENTS IN PARTSIVAL}

partsival features several areas of performance improvement, beyond the plain computational component. In this section, the most important features are explained.

\subsection{Dynamic Floating Point Precision}

partisval features the ability to decide whether to use single, double or mixed floating point precision after compile time [1]. This allows to assess for the precision required by each application. The idea is that simulations for absolute statements like predictions of system behaviour have to be in double precision. whereas comparative, relative statements as used in optimization campaigns can often be run in single
precision. However, it was found, that single precision performs just fine even for some absolute predic-
tions in terrestrial gravity. This can be seen in the domparison of a single wheel simulation in terrestrjal
gravity shown in Figure 3 . Nonethetess, it should be noted that with decreasing gravity forces become
smaller and floating point errors become more pronounced. Thus, indeterminism due to parallelization can happen in milli-g environments [12].
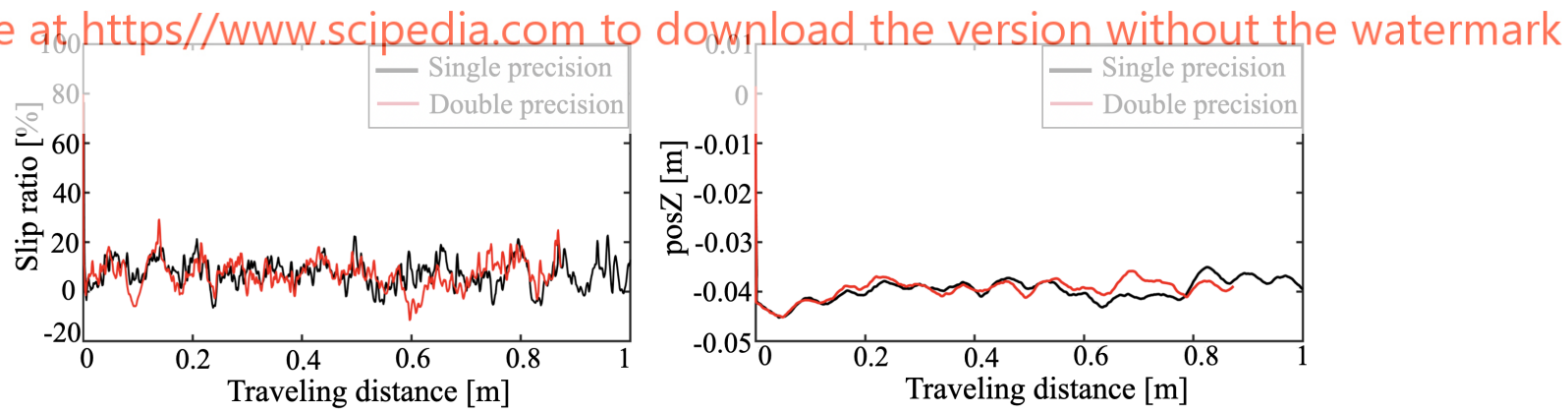

Figure 3: Single precision vs Double precision (left: slip ratio, right: sinkage)

Mixed precision uses the idea to combine single and double floating point precision in cases where the rounding errors get significant or the numeric problems of cancelation and absorption arise. As the main calculations are done in single precision the performance can be held as high as possible, while critical operations can be done in double precision with conversion of the results to single precision thereafter. In 
partsival this mechanism is mainly used in the integration algorithm, but will be included in the contact models in the future to allow for faster computation even in lower gravity.

\subsection{Jolt-based Integration Schemes}

In the discrete element method, simple explicit integration schemes are still the state of the art. The biggest downside of these schemes is the connection between stability, time step size and eigen frequency of the system, which requires small step sizes. Hence, (semi-)implicit integration schemes often yield faster and more stable simulations [16]. But these advantages come at a cost. The semi-implicit schemes are mostly implemented as predictor corrector algorithms. Hence, they need a repeated contact detection step in every corrector step.

In partsival, a special semi-implicit integrator has been developed that omits these renewed contact detection steps [3]. It uses the assumption that for a stable system, the fourth derivative of the position, i.e. the jolt will not change significantly during subsequent time steps:

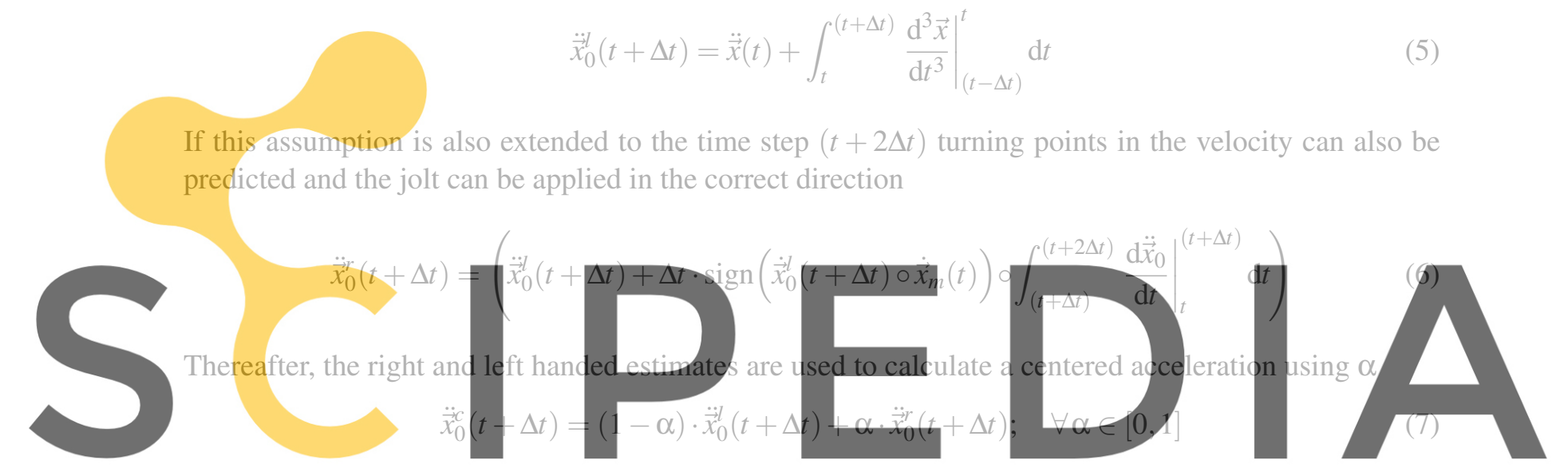

and applying the result to the well known BEEMAN [17] scheme thereafter to get velocity $\dot{\bar{x}}_{0}^{c}(t+\Delta t)$

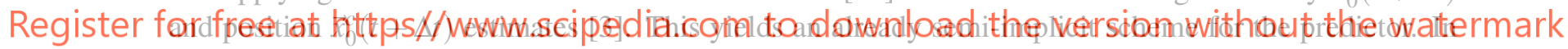

the corrector loop the future acceleration is still estimated. However, the predicted estimate is improved

based on the force law used, in this case by linearization of the elastic contact:

$$
\ddot{\vec{x}}_{n}^{c}(t+\Delta t)=\ddot{\vec{x}}_{m}(t)+\xi \int_{\vec{x}_{m}(t)}^{\vec{x}_{n-1}(t+\Delta t)}\left(\nabla \circ \ddot{\vec{x}}_{\vec{x}_{m}(t-\Delta t)}^{\vec{x}_{m}(t)}\right) \mathrm{d} \vec{x} ; \quad \forall \xi \in[0,1]
$$

with $\xi$ weighting the linearized force-law. The corrector loop is usually run for $i \in[1,3]$ to yield sufficient accuracy. After the loop is completed the missing velocity is computed only once, while acceleration and position are updated in every corrector step. As all the future accelerations in this scheme were purely predicted, the contact detection of the next step is used to assess the error between the estimate and the true accelerations. This error is then fed into a control algorithm that tries to minimize the error $\varepsilon=\ddot{\vec{x}}_{\mathrm{cd}}(t)-\ddot{\vec{x}}_{m}(t)$ using the time step size as control actuator. Further information on the scheme and step size control is given in [3]. Given the results in [3] as well as more recent tests, the computation performance is increased by a factor of two to four compared to a conventional NEWMARK- $\beta$ integrator. The performance gain over explicit schemes is hard to judge, as it depends on the problem to be simulated and the stability conditions the gain may vary significantly. 


\subsection{Boundary Conditions}

Boundary conditions are essential to every DEM simulation, but can also be used to speed up computation significantly. Thereby, the proper choice as well as special boundaries are discussed within the next sections.

\subsubsection{General Boundaries}

Generally, the soil bed is to be chosen sufficiently large to avoid boundary effects on the soil flow and sizing constraints for the simulation domain depending on loads and tool dimensions can be derived [9]. Multiple boundary conditions are available in order to match experiments or save computational effort. Container walls have their own coefficient of friction for stick and slip and are modeled as meshed surfaces. These smooth boundaries are used whenever a physical boundary is modeled. However, if the container and thus the volume of soil is much larger than the region of influence, stationary layers of particles can directly build up a border of the domain, being rough surfaces. These consist of several layers of fixed particles that restrict soil motion and ensure homogeneous friction.

Symmetry conditions are another useful type of boundary that can be used to save computational effort by exploiting symmetry in the simulated geometry. In partsival, both plane and rotation symmetry conditions can be used. As an extreme case of both conditions, a 2D model is resulting form infinitely small symmetric sections.

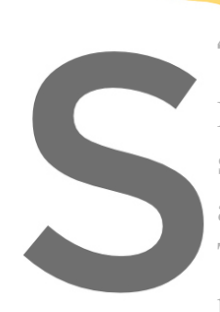

\subsubsection{Dynamic Boundaries}

Even modern GPUs are only able to compute several thousands of particles in parallel, whereas a usuan
simulation scenario consists of several tens or hundreds of thousand particles. Dynamic boundaries are
able to substantially lower the number of active particles and thus speed up contact detection significantiy.
This is due to the fact that the influence of a tool only reaches finite dimensions, yhereas outside of these
region, forces and stresses remain almost untouched. The basic theory behind dynamic boundaries is explained alongside applicable influence regions for

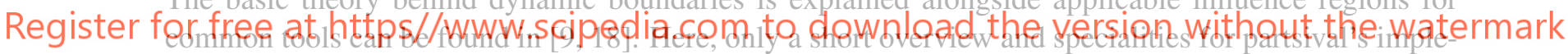

mentation shall be given. Dynamic boundaries are a special version of rough boundaries. Thereby, the boundary is moved along with the windows around the tool manipulating the soil. This is done by loading and deleting particles which are in or out of the influence region respectively. The outermost layers of particles (at least two) of this region are fixed with 0 DoF in order build up the boundary itself. They are also included in force calculation and their velocity is set to zero once they are freed. A dynamic boundary fixed to a wheel is shown in Figure 4.

In order to perform time efficient simulations, all particles which are outside of the influence region are already existent in the GPU memory and are held even after "deletion". This avoids communication and loading of particles from CPU space or even hard drive, but is less memory efficient. However, given the large amount of memory of current graphics cards, the additional memory needed is affordable and large enough for most simulations with up to several millions of particles.

\section{Applications \& Validation}

In this section, two applications in planetary exploration will show the applicability of partsival and the special measures for large scale simulation campaigns. 

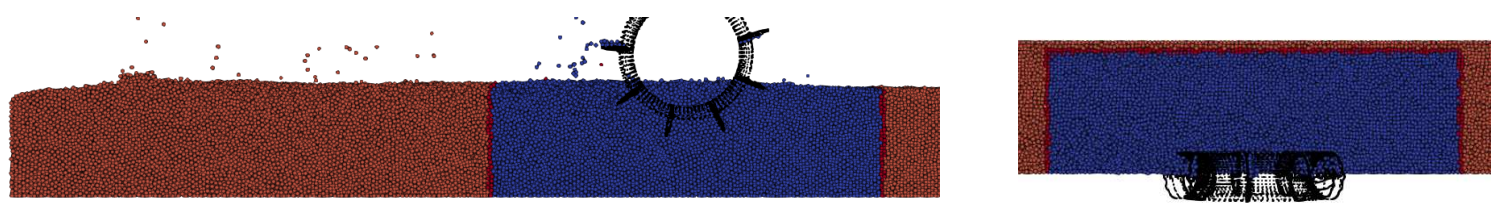

Figure 4: Dynamic boundaries (side and top view with symmetry condition), blue particles are free 6 DoF, dark red ones are 0 DoF boundary particles still in contact detection and light red ones are non existent for the simulation

\subsection{Validation}

In this study, three types of validation were carried out to examine the accuracy and applicability of the DEM simulation. One of them is the angle of repose test, and the other two are single wheel tests.

\subsubsection{Angle of Repose Tests}

As the simple preliminary validation of the DEM simulation, the angle of repose tests were implemented in both simulation and experiment [19]. In the experiment, the soil called RMCS14, which is a mixture of Olivine sands with different grain size ranges, was used [20].

The sand piles in both the simulation and experiment are shown in Figure 5. These figures indicate that the shape of the sand piles is nearly identical. The angle of repose measured from images is $29.0^{\circ}$ in the simulation and $30.4^{\circ}$ in the experiment. These preliminary tests indicate that the shape of the sand piles

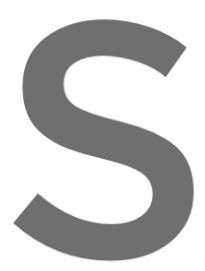
and the angle of repose $i$
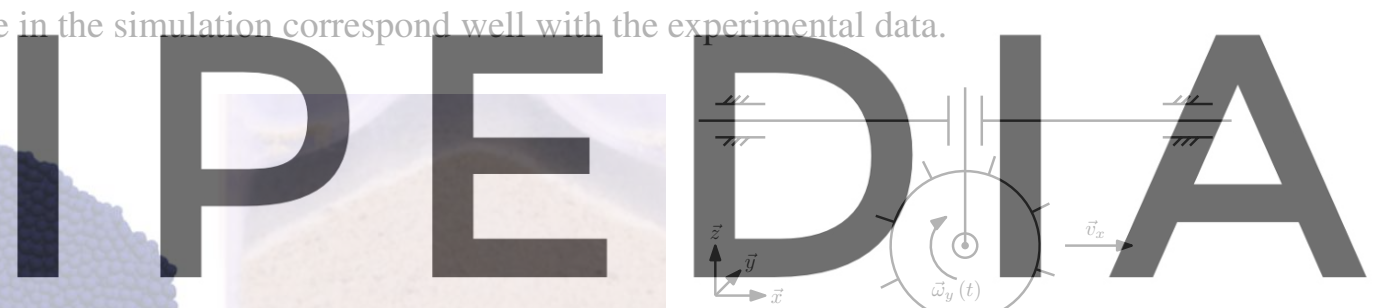

Register for free at https//www.scipedia.com to download the

Figure 5: Sand pile (left: simulation, right: experiment[19])

Figure 6: Single wheel simulation model with free slip conditions

\subsubsection{Single Wheel Tests in 3D}

The validation of the DEM simulation for the single wheel tests is necessary to evaluate the wheel performance. In the 3D-DEM simulation, we can investigate the effect of complex wheel shapes on the wheel performance. This is beneficial to optimize the wheel shape for future exploration missions. For the validation of 3D-DEM, the simulation result was compared with the single wheel experiments using TROLL, which is a robot-based testbed at DLR [20]. In the tests, RMCS14 was also used, and the particle parameters for the simulation were determined based on this soil. The wheel configuration is the same setup with the experimental one, and the wheel travels with a constant angular velocity under free slip condition. The schematic diagram of the single wheel simulation is illustrated in Figure 6.

In this validation, we focus on the wheel slippage and sinkage since excessive slippage and sinkage can cause entrapment of the wheel in the soft soil and are thus important performance measures for the 
wheels. In Figure 7, the black lines show experimental data and the red lines show the simulation results. The range painted in blue shows $3 \sigma$ confidence intervals of the experiments, where $\sigma$ is the standard deviation of the experiments. For the slip ratio, the outcome between the simulation and experiment shows good agreement. For the sinkage, some places deviate from $3 \sigma$, but the value is still close to the experimental data. Thus, the accuracy of the DEM simulation in terms of slip ratio and sinkage is enough to evaluate the wheel performance.
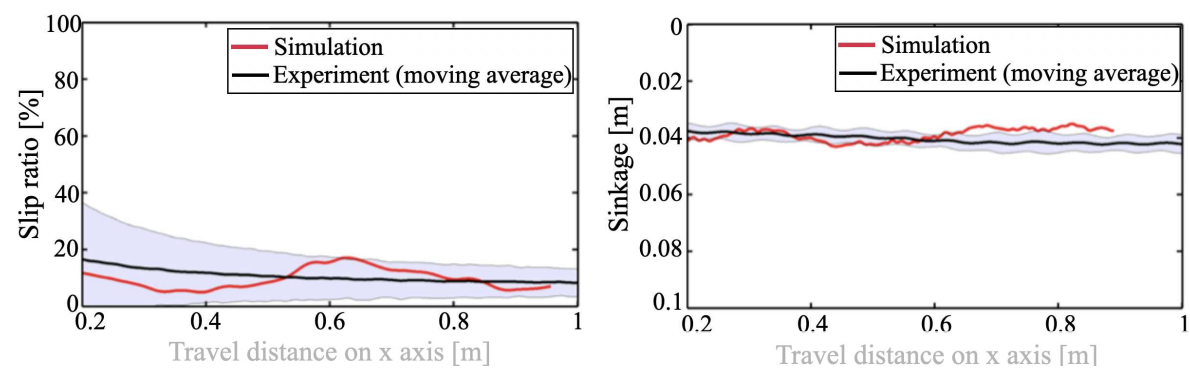

Figure 7: Validation of the single wheel tests (left: slip ratio, right: sinkage)

\subsubsection{Single Wheel Tests in 2D}

The visualization of soil flow and deformation is beneficial to understand wheel-soil interaction mechan-

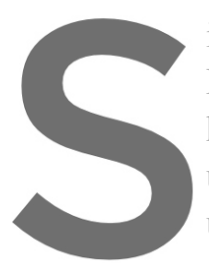

ics. Thus, the DEM simul

For the observation of

haviour of particles at hig

used, but the movement

ulation results are compa

analysis in Tohoku University, the wheel configura
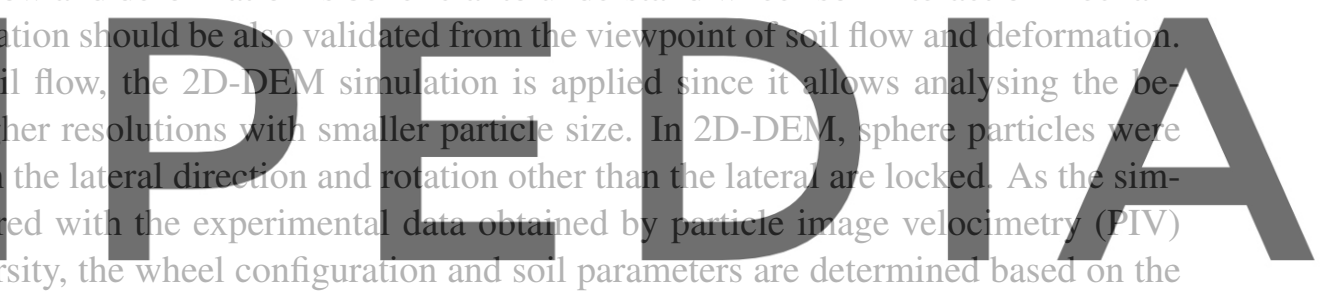

experimental setup [21]. To determine the particle parameters, partsival allows us to conduct many sim-

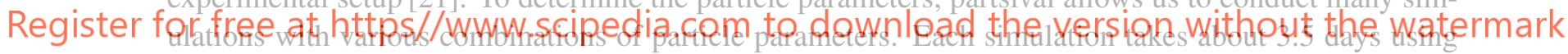

Tesla V100 GPUs.

Figure 8 shows the ruts formed by the wheel and soil fiow velocity fieid beneath the wheel. The wheei travels from the left to right on each figure. The ruts are also observed in the experiments as well as the simulation. For the velocity filed of the soil particles obtained from DEM simulation, the vectors show the magnitude of the soil velocity and its direction is the flow direction. The soil flow toward the back of the wheel was observed, and this flow mainly occurs that the grouser pushes the soil to the back of the wheel. The wheel obtains the traction as a reactive force pushing this soil backward. This soil behaviour were also observed in the PIV experiment conducted in [21]. These results indicate that the applicability of the DEM simulation to analyze the soil flow and deformation and wheel-traveling performance.
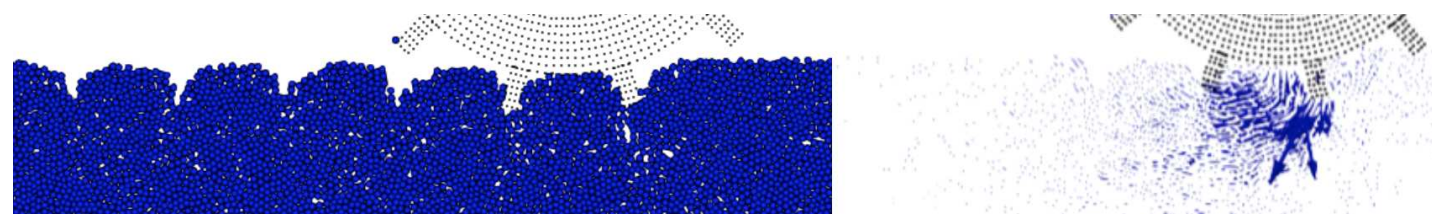

Figure 8: 2D simulation (left: ruts formed by the wheel, right: soil velocity field) 


\subsection{Wheel Optimization Campaign}

A recent application where partsival was successfully applied is the wheel design for the Martian Moon Exploration (MMX) rover [22]. Its mission goals are the demonstration of wheeled locomotion in lowgravity, in-situ science with dedicated instruments and scouting, i.e. hazard avoidance, for the spacecraft's landing. The rover will have four aluminium wheels that need to be designed for the surface conditions of Phobos. A current locomotion prototype is shown in Figure 9.

Phobos provides a difficult environment for a wheeled rover due to low gravity and unknown regolith conditions. The effective surface acceleration of Phobos is $3 \mathrm{~mm} \mathrm{~s}^{-2}$ to $6.8 \mathrm{~mm} \mathrm{~s}^{-2}$ [23]. For wheel design a value of $6 \mathrm{~mm} \mathrm{~s}^{-2}$ was assumed, which is about $1 / 2000$ of the respective value on Earth. For the mostly unknown regolith conditions, a worst-case of soft, cohesion-less soil was defined [23] and implemented in a DEM simulation to optimize wheel traction.

Previous design efforts for the MMX wheels were based on a simulation study for Mars rovers [10] and a "one-factor-at-a-time" optimization study for Phobos [11]. To expand on these efforts, a DEM based Bayesian Optimization [24] was implemented. The wheel shape is parametrized using four parameters: grouser height relative to the wheel diameter, grouser number, grouser chevron angle and rim curvature. As stowage space on the spacecraft is limited and larger wheels are well documented to perform better on soft soils [11], the maximum possible outer wheel diameter was chosen as a constraint. Each simulations covers 1.5 wheel revolutions in $50 \mathrm{~s}$ on a soil bed with more than 260000 particles. The simulations are run in partsival on Nvidia V100 GPUs and take about $20 \mathrm{~h}$ each. Three scenarios were chosen to cover the rovers driving conditions during operations: Driving forward on flat ground, driving forward up a 10 degree slope and drivi on the covered distance $x$ shown in Figure 10 [11] The optimization was run for 115 fitness evaluation It has seven grousers of $6 \mathrm{~cm}$ height. a rim curvature It performs $8 \%$ better than the intermediate design
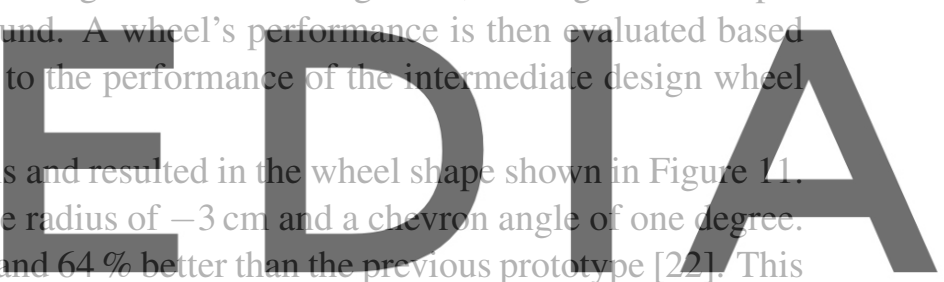

large performance increase was enabled by the ability to efficiently run simulations on GPUs in parallel.

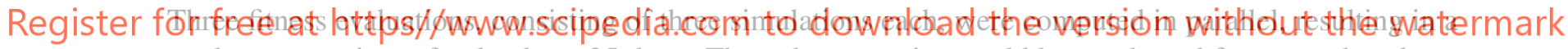
total compute time of only about 35 days. Thus, the campaign could be conducted fast enough to keep the mission's tight schedule even though limited computation hardware and long simulation times have been used.

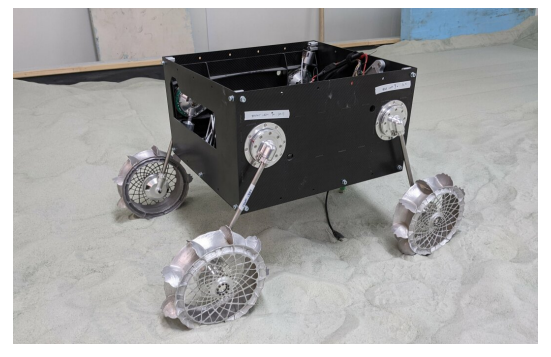

Figure 9: The MMX rover prototype

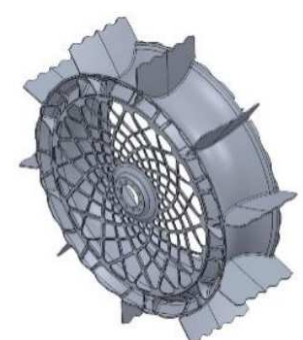

Figure 10: Intermediate wheel Figure 11: The optimized wheel design [11]

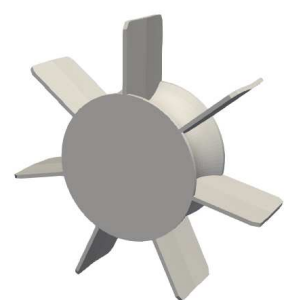

shape 


\section{CONCLUSION \& FURTHER WORK}

In this paper, partsival, a DEM simulation tool especially developed for large scale simulations and campaigns in planetary exploration, has been presented. Recent advances in partsival have been explained and numerical considerations regarding floating point precision have been made. Furthermore, contact models that help to keep computational cost low while gaining high accuracy have been introduced alongside boundary conditions especially suited to speed up simulations.

Using two applications, a large scale optimization campaign and an ongoing in-depth validation, the applicability of the approach to these large scale problems is demonstrated. The validation shows, that the search for the correct parameter combinations and repeated model adaptions can be efficiently done in partsival even for complex models like wheel simulations. Furthermore, the optimization campaign shows that even though very limited computation hardware has been used, a large scale campaign can be finished within a month and thus in the schedule of an actual space mission. The validation results indicate that the DEM simulation using partsival is valid not only for the evaluation of wheel performance but also for the analysis of soil flow.

This simulation tool will be also used to analyse the microscopic behaviour of soil such as stresses acting on particles. In the future, the focus will be on enhancing the rolling models by rounded edges and even more complex particles shapes, while keeping the models as efficient as possible. A further goal is to improve the contact detection algorithms, which are currently the most costly part of the simulations. Additionally, ongoing validation will ensure that partsival is always compliant with the high demand for accuracy in planetary exploration.

\section{REFERENCES}

[1] R. Lichtenheldt, S. Kerler, A. Angerer, and W. Reif, "partsival - collision-based particle and many-body simulations on GPUs for planetary exploration systems," in 5th Joint International Conference on Multibody System Dynamics, 2018.

[2] R. Lichtenheldt and B. Schäfer, "Planetary Rover Locomotion on soft granular Soils - Efficient Adaption of the rolling Behaviour of nonspherical Grains for Discrete Element Simulations," in 3rd International Conference on Particle-Based Methods, pp. 807-818, 2013.

[3] R. Lichtenheldt, "A stable, implicit time integration scheme for discrete element method and contact problems in dynamics," in V International Conference on Particle-based Methods - Fundamentals and Applications, vol. 5, pp. 297-308, 2017.

[4] P. A. Cundall and O. D. L. Strack, "A discrete numerical model for granular assemblies," Géotechnique, vol. 29, pp. 47-65, 1979.

[5] S. Agarwal, C. Senatore, T. Zhang, M. Kingsbury, K. Iagnemma, D. I. Goldman, and K. Kamrin, "Modeling of the interaction of rigid wheels with dry granular media," Journal of Terramechanics, vol. 85, pp. 1-14, 2019.

[6] M. A. Knuth, J. B. Johnson, M. A. Hopkins, R. J. Sullivan, and J. M. Moore, "Discrete element modeling of a mars exploration rover wheel in granular material," Journal of Terramechanics, vol. 49, pp. 27-36, 2012.

[7] W. Smith, D. Melanz, C. Senatore, K. Iagnemma, and H. Peng, "Comparison of discrete element method and traditional modeling methods for steady-state wheel-terrain interaction of small vehicles," Journal of Terramechanics, vol. 56, pp. 61-75, 2014.

[8] J. B. Johnson, A. V. Kulchitsky, P. Duvoy, K. Iagnemma, C. Senatore, R. E. Arvidson, and J. Moore, "Discrete element method simulations of mars exploration rover wheel performance," Journal of Terramechanics, vol. 62 , pp. 31-40, 2015. 
[9] R. Lichtenheldt, Lokomotorische Interaktion Planetarer Explorationssysteme mit weichen Sandböden - Modellbildung und Simulation. PhD thesis, Ilmenau University of Technology, 2016.

[10] S. Ono, "Evaluation of Planetary Rover Wheel Performance on Sloped Loose Soil Based on Discrete Element Method," Master's thesis, Tohoku University, 2019.

[11] F. Buchele and R. Lichtenheldt, "Multi-parameter rover wheel and grouser optimization for deployment in phobos mili-g environment," in Proceedings of the International Symposium on Artificial Intelligence, Robotics and Automation in Space (i-SAIRAS), 2020.

[12] F. Buchele, R. Lichtenheldt, and L. Stubbig, "Numerics of discrete element simulations in milli-g environments: Challenges and solutions," in 7th International Conference on Particle-based Methods, 2021.

[13] R. Lichtenheldt, "A novel systematic method to estimate the contact parameters of particles in discrete element simulations of soil," in 4th International Conference on Particle-based Methods, pp. 430 - 441, 2015.

[14] M. Obermayr, C. Vrettos, P. Eberhard, and T. Däuwel, "A discrete element model and its experimental validation for the prediction of draft forces in cohesive soil," Journal of Terramechanics, vol. 53, pp. 93-104, 2014.

[15] R. Loy, “Modellierung von Kohäsionseffekten für planetares Regolith in Partikelsimulationen.” Bachelor thesis, Nuremberg Institute of Technology, 2019.

[16] F. Fleissner, Parallel Object Oriented Simulation with Lagrangian Particle Methods. PhD thesis, Universität Stuttgart, 2010.

[17] D. Beeman, "Some multistep methods for use in molecular dynamics calculations," Journal of Computational Physics, vol. 20, pp. 130-139, 1976.

[18] R. Lichtenheldt, “Covering shock waves on Mars induced by InSight's HP3-mole - Efficient co-simulation using DEM and multi-domain dynamics," in 7th International Conference on Computational Methods for Coupled Problems in Science and Engineering, 2017.

[19] S. Ono, R. Lichtenheldt, and K. Yoshida, "Parametric influnces on the behaviour of planetary regolith using dem simulations," in Proceedings of the International Symposium on Artificial Intelligence, Robotics and Automation in Space (i-SAIRAS), 2018.

[20] F. Buse, T. Bellmann, R. Lichtenheldt, and R. Krenn, “The DLR Terramechanics Robotics Locomotion Lab," in Proceedings of the International Symposium on Artificial Intelligence, Robotics and Automation in Space (i-SAIRAS), 2018.

[21] S. Ono, S. Namikawa, and K. Yoshida, "Analysis of Soil Deformation and Wheel Traction on Loose Terrain Using PIV,” in IEEE Aerospace Conference, 2020.

[22] H.-J. Sedlmayr, S. Barthelmes, R. Bayer, W. Bertleff, M. Bihler, F. Buse, M. Chalon, D. Franke, F. Ginner, V. Langofer, et al., "MMX - Development of a Rover Locomotion System for Phobos," in 2020 IEEE Aerospace Conference, pp. 1-10, 2020.

[23] S. Tardivel and J. Biele, "Phobos environment requirement document for the MMX rover mission," tech. rep., CNES \& DLR, 2019.

[24] P. I. Frazier, “A tutorial on bayesian optimization,” arXiv preprint arXiv:1807.02811, 2018. 